OPEN ACCESS

Edited by:

Rong Tian,

Sichuan University, China

Reviewed by:

Laurence Gluch,

The Strathfield Breast

Centre, Australia

Zhongxiang Ding,

Hangzhou First People's

Hospital, China

*Correspondence:

Zhi-Cheng Li

zc.li@siat.ac.cn

Desheng Sun

szdssun@163.com

${ }^{\dagger}$ These authors have contributed equally to this work

Specialty section

This article was submitted to

Cancer Imaging and Image-directed Interventions,

a section of the journal

Frontiers in Oncology

Received: 01 September 2019 Accepted: 13 January 2020

Published: 31 January 2020

Citation:

Sun Q, Lin X, Zhao Y, Li L, Yan K, Liang D, Sun D and Li Z-C (2020)

Deep Learning vs. Radiomics for

Predicting Axillary Lymph Node Metastasis of Breast Cancer Using Ultrasound Images: Don't Forget the

Peritumoral Region.

Front. Oncol. 10:53.

doi: $10.3389 /$ fonc. 2020.00053

\section{Deep Learning vs. Radiomics for Predicting Axillary Lymph Node Metastasis of Breast Cancer Using Ultrasound Images: Don't Forget the Peritumoral Region}

\author{
Qiuchang Sun ${ }^{1 \dagger}$, Xiaona Lin ${ }^{2 \dagger}$, Yuanshen Zhao ${ }^{1}$, Ling $\mathrm{Li}^{3}$, Kai Yan ${ }^{1,4}$, Dong Liang ${ }^{1}$, \\ Desheng Sun ${ }^{2 *}$ and Zhi-Cheng $\mathrm{Li}^{1 *}$ \\ ${ }^{1}$ Institute of Biomedical and Health Engineering, Shenzhen Institutes of Advanced Technology, Chinese Academy of \\ Sciences, Shenzhen, China, ${ }^{2}$ Department of Ultrasonic Imaging, Peking University Shenzhen Hospital, Shenzhen, China, \\ ${ }^{3}$ Ultimage Lab, Suzhou, China, ${ }^{4}$ Peng Cheng Laboratory, Shenzhen, China
}

Objective: Axillary lymph node (ALN) metastasis status is important in guiding treatment in breast cancer. The aims were to assess how deep convolutional neural network (CNN) performed compared with radiomics analysis in predicting ALN metastasis using breast ultrasound, and to investigate the value of both intratumoral and peritumoral regions in ALN metastasis prediction.

Methods: We retrospectively enrolled 479 breast cancer patients with 2,395 breast ultrasound images. Based on the intratumoral, peritumoral, and combined intra- and peritumoral regions, three CNNs were built using DenseNet, and three radiomics models were built using random forest, respectively. By combining the molecular subtype, another three CNNs and three radiomics models were built. All models were built on training cohort (343 patients 1,715 images) and evaluated on testing cohort (136 patients 680 images) with ROC analysis. Another prospective cohort of 16 patients was enrolled to further test the models.

Results: AUCs of image-only CNNs in both training/testing cohorts were 0.957/0.912 for combined region, $0.944 / 0.775$ for peritumoral region, and 0.937/0.748 for intratumoral region, which were numerically higher than their corresponding radiomics models with AUCs of 0.940/0.886, 0.920/0.724, and 0.913/0.693. The overall performance of image-molecular CNNs in terms of AUCs on training/testing cohorts slightly increased to 0.962/0.933, 0.951/0.813, and 0.931/0.794, respectively. AUCs of both CNNs and radiomics models built on combined region were significantly better than those on either intratumoral or peritumoral region on the testing cohort $(p<0.05)$. In the prospective study, the CNN model built on combined region achieved the highest AUC of 0.95 among all image-only models. 
Conclusions: CNNs showed numerically better overall performance compared with radiomics models in predicting ALN metastasis in breast cancer. For both CNNs and radiomics models, combining intratumoral, and peritumoral regions achieved significantly better performance.

Keywords: breast cancer, deep learning, radiomics, axillary lymph node metastasis, breast ultrasound, peritumoral region

\section{INTRODUCTION}

Breast cancer is the leading malignancy in females (1). Axillary lymph node (ALN) metastasis status is one of the most important factors in guiding treatment decision making in breast cancer (2). Traditionally, the nodal status was assessed by surgical methods such as sentinel lymph node biopsy (SLNB) and axillary lymph node dissection (ALND) (3). According to the guideline from American Society of Clinical Oncology, SLNB is considered to have a high overall accuracy ranging from 93 to $97.6 \%$ with a relatively low false negative rate (FNR) ranging from 4.6 to $16.7 \%$ in detecting axillary metastasis (4). However, these surgical approaches have been considered controversial due to the invasiveness, potential complications, and possible overtreatment (3-6).

Ultrasound is a widely-used tool in breast cancer assessment as it is non-invasive, radiation-free, real-time and well-tolerated in women. Previous studies have shown that axillary ultrasound (AUS) may provide useful information relevant to ALN status in breast cancer (7). However, AUS alone has moderate sensitivity and may not be a reliable predictor for nodal metastasis $(7,8)$. Recently, imaging-based machine learning approaches have been demonstrated promising in cancer diagnosis. There are two most popular machine learning approaches: radiomics analysis and convolutional neural networks (CNN). Radiomics analysis relies on a pipeline including extraction of numerous handcrafted imaging features, followed by feature selection and machine learning-based classification. Handcrafted radiomics features extracted from the breast tumor area have been demonstrated predictive in ALN metastasis, with FNRs ranging from 13.9 to $25 \%(9,10)$. However, handcrafted features are limited to the current knowledge of medical imaging, which may limit the potential of the predictive model. Deep learning improves this handcrafted pipeline by automatically learning discriminative features directly from images. Recent studies have shown that deep CNN-based approaches can achieve state-of-the-art performance in lesion detection and cancer diagnosis (11-13). To our knowledge, no studies have assessed breast ultrasound-based $\mathrm{CNN}$ in predicting ALN status for breast tumor.

Most studies have focused on mining predictive imaging features within the tumor, while the surrounding tissues were ignored. Previous evidence has shown that the peritumoral region-the tumor-adjacent parenchyma immediately surrounding the tumor mass-may offer valuable outcomeassociated information (14-16). Two recent studies have demonstrated that handcrafted imaging features from peritumoral region in Dynamic Contrast-Enhanced MRI
(DCE-MRI) are associated with sentinel lymph node metastasis (9) and pathological complete response to neoadjuvant chemotherapy (17) in breast cancer. Here, we hypothesize that deep CNN built based on intra- and peritumoral regions in breast ultrasound could provide relevant information in predicting ALN status. We are interested in comparing the performance of deep CNNs and radiomics models. Additionally, breast cancer can be classified into different molecular subtypes with distinct prognosis and respond differently to specific therapies (18). Therefore, we further assessed if deep CNNs or radiomics models combining imaging features and molecular subtypes could offer improved accuracy.

In this hypothesis-driven study, we first developed deep CNNs and radiomics models based on intratumoral, peritumoral, and combined regions in breast ultrasound images for predicting ALN metastasis. We then aimed to find out how on each region deep CNNs performed compared with radiomics models.

\section{MATERIALS AND METHODS}

\section{Study Population}

The study was approved by the Ethics Committee of Peking University Shenzhen Hospital (PUSH). Informed consent was waved from all patients by the ethics committee of PUSH. From the pathology and radiology databases in PUSH, a retrospective search was performed to recruit female patients with breast cancer between January 2016 and December 2018. The inclusion criteria were patients (1) with histologically-confirmed primary breast cancer, (2) with pretreatment breast ultrasound images, (3) with known ALN metastasis status determined by final histopathology, (4) with known molecular subtypes, and (5) without neoadjuvant chemotherapy prior to SLNB or ALND. The exclusion rules were that patients (1) with very small region of interest in the ultrasound images ( $<100$ pixels) and (2) without SLNB or ALND. Finally, 479 patients with 479 breast tumors (136 positive and 343 negative ALNs) were included in this study. This cohort was randomly divided into a training cohort of 359 patients and a testing cohort of 120 patients at a ratio $3: 1$. The patient recruitment pathway was shown in Figure $\mathbf{S} 1$.

The baseline clinical and histopathological data were derived from patient medical records, including age, histological grade, immunohistochemistry (IHC) results and ALN status (positive or negative). According to the 2017 St Gallen International Expert Consensus, each patient was classified into one of four molecular subtypes: human epidermal growth factor receptor-2 (HER2) positive, triple-negative, Luminal A, and Luminal B (18). The status of HER2, ER, progesterone receptors (PR) and Ki-67 was 
assessed by IHC. Based on the IHC results, the subtype can be determined.

\section{Ultrasound Image Acquisition}

The breast ultrasound examinations were performed by breast radiologists in our center using the Hitachi Ascendus ultrasound system equipped with $13-3 \mathrm{MHz}$ linear array transducers. The examinations and assessments were conducted according to the 5th edition of Breast Imaging Reporting and Data System (BIRADS) presented by American College of Radiology (ACR) (19). The parameters were set as follows: depth, $4-5 \mathrm{~cm}$; brightness gain, 10-25 dB; dynamic range, $70 \mathrm{~dB}$; frame rate, 26 frame per second. Patients were placed in supine or lateral position. The field of view was set to have the pectoralis muscle at the deepest aspect of the image. The focal zone was adjusted to be centered at the lesion. Ultrasound images were acquired and documented into the Picture Archiving and Communication Systems (PACS). For each lesion, five images were selected from PACS by a breast radiologist (XL with 5 years' experience in breast radiology) and used in our study according to the following scheme: (1) an image along the longest axis of lesion. (2) an image orthogonal to the first image. (3) three images at other angles where the lesion was clearly presented. The five images together represented the ultrasonographic features of a 3D lesion from different angels. For all 479 patients, we finally obtained 2395 images in total, including 1715 images (343 patients) in the training cohort and 680 images (136 patients) in the testing cohort.

\section{ROI Delineation}

The tumor region in each ultrasound image was manually delineated using the ITK-SNAP software (http://www.itksnap. org) by one radiologist (XL) who were blinded to the clinical and histopathological data of patients. A second breast radiologist (DS with 12 years' experience in breast radiology) reviewed all the delineations. Any disagreement between the two raters was resolved by discussion and consensus. The peritumoral regions were obtained by dilating the delineated tumor contour by $\sim 5 \mathrm{~mm}$ based on a standard morphological dilation operation using an inhouse program implemented in Matlab 2016b (MathWorks, Natick, MA). For each ultrasound slice, three region of interest (ROI) images were finally obtained: the intratumor ROI, the peritumor ROI, and the combined ROI that merged the intratumor and the peritumoral regions. Examples of ultrasound slices overlapped with intratumoral and peritumoral ROIs for two patients were shown in Figure 1.

\section{Deep Learning With DenseNet}

Deep CNN can automatically learn discriminative features from imaging data by stacking multiple convolutional layers. Among different CNN variants, densely connected convolutional network (DenseNet) has shown superior classification performance as it strengthens feature propagation while reduces parameter number (20). This is accomplished by connecting each layer to every other layer in a feed-forward fashion with less computational complexity. Here, our model was built based on the standard DenseNet-121 (20). All ROI images were resized into $224 \times 224$. The resized ROI images were used as input and transformed through the chained convolutional layers, yielding a class probability vector as the prediction results. The network was trained from scratch with cross entropy loss function and Adam optimizer with a learning rate of 0.0001 , a batch size of 16 , and a regularization weight of 0.0001 . In the training cohort, data augmentation approaches including random rotation, random shear and random zoom were employed before the training procedure to avoid possible overfitting. The network was implemented on Keras (https:// keras.io/) with the TensorFlow library as the backend (https:// www.tensorflow.org/). The architecture of the image-only CNN network was shown in Figure 2. The details of the convolutional network implementation can be found in Table S1.

\section{Deep Learning-Based Predictive Model Building}

For predicting the nodal status, three image-only CNN models, including the intratumoral $\mathrm{CNN}$, the peritumoral $\mathrm{CNN}$ and the combined-region CNN, were built with the DenseNet based on the intratumor ROI images, the peritumor ROI images, and the combined ROI images, respectively. Furthermore, three corresponding image-molecular models were also built based on the DenseNet by using both ROI images and molecular subtype information as the network input. Specifically, the molecular subtype information was incorporated as input to the fullyconnected layers of the DenseNet, as shown in Figure 2.

\section{Radiomics Feature Extraction and Selection}

For each ultrasound slice, 104 radiomics features were extracted from each of the three ROI areas by using an open-source toolbox named Pyradiomics (https://pyradiomics.readthedocs. io) (21). Three groups of features were extracted, including shape features, intensity features, and texture features, as summarized in Table S2. Eleven shape features describing the geometric characteristics of the ROI were extracted. Eighteen intensity features describing the first-order distribution of the ROI intensities were extracted. Seventy-five texture features were computed to describe the patterns, or the high-order distributions of the ROI intensities with five different methods, including the gray-level co-occurrence matrix (GLCM), graylevel run length matrix (GLRLM), gray level size zone matrix (GLSZM), gray level dependence matrix (GLDM), and neighborhood gray-tone difference matrix (NGTDM). The detailed definitions of the radiomics features used can be found in two articles $(22,23)$. Having high-dimensional radiomics features, feature selection is required to reduce the dimension and avoid overfitting. Here an efficient machine learning-based wrapper algorithm, Boruta, was used to select a subset of features that were relevant to the prediction outcome (24). Boruta evaluated feature relevance iteratively by comparing the importance of original features with that achieved by artificially added random features, yielding an all-relevant subset of features that was considered optimal for the classification task. Here we used the R package Boruta for Boruta feature selection. 

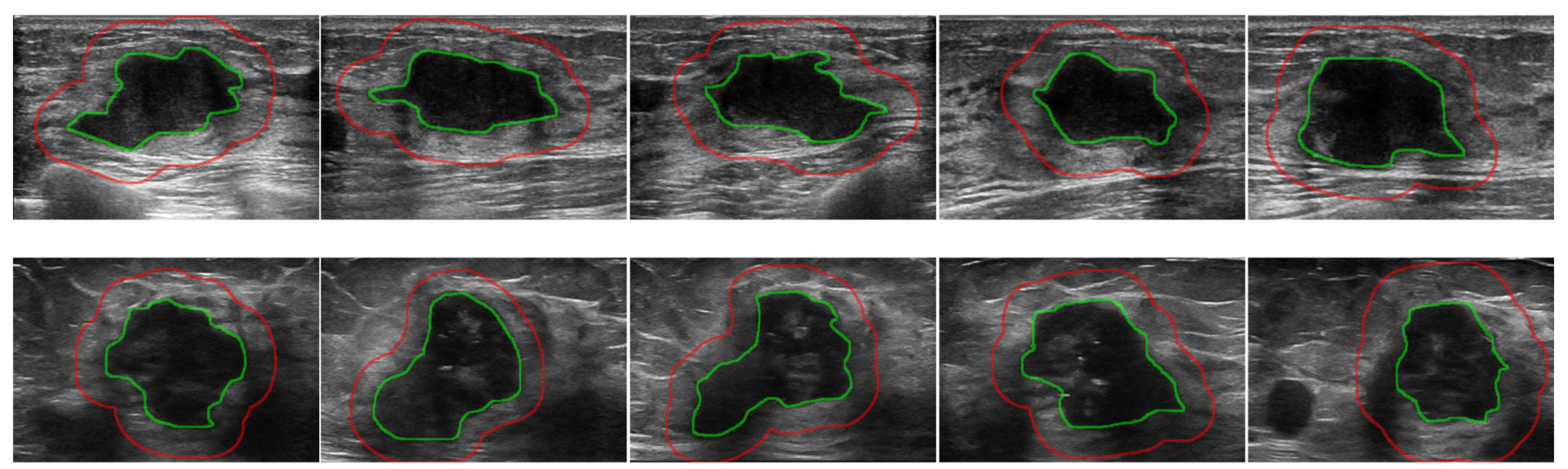

FIGURE 1 | Examples of ultrasound slices overlapped with intratumoral regions (green) and peritumoral regions (red) from two patients. (Top) A patient with positive ALN. (Bottom) A patient with negative ALN.

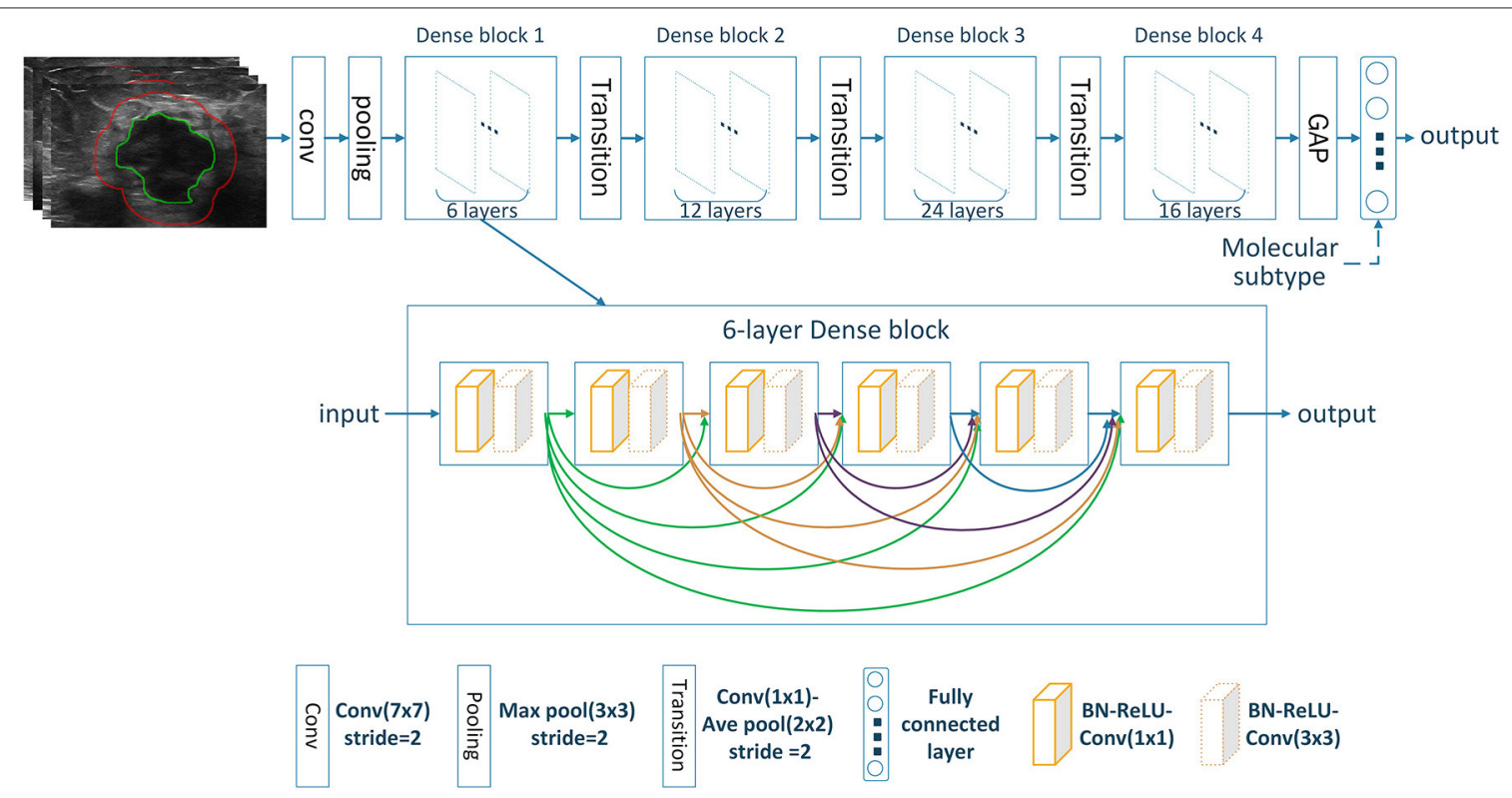

FIGURE 2 | The architecture of the deep CNN used in our study.

\section{Radiomics-Based Predictive Model Building}

Based on the selected radiomics features, three image-only radiomics models were built using random forest algorithm (25) based on the intratumor ROI, the peritumor ROI, and the combined ROI, respectively. Correspondingly, three imagemolecular radiomics models were also built using random forest by integrating ROI images and molecular subtype information as the input. After testing different settings, the tree number of all random forest classifiers was set to 300 . Gini index was used as importance measure (26). The $\mathrm{R}$ package randomForest was used for random forest classification.

\section{Statistical Analysis}

The difference in age, histological grades and molecular subtypes between training and testing cohorts was assessed with $\chi^{2}$ test or Wilcoxon rank-sum test, where appropriate.
All 12 prediction models (3 image-only CNNs, 3 imageonly radiomics models, 3 image-molecular $\mathrm{CNNs}$ and 3 image-molecular radiomics models) were trained on the training cohort and evaluated on the testing cohort. Because each tumor had five ultrasound images, there were five corresponding prediction outcomes in the form of class probabilities. Among them, the median probability was chosen as the final prediction of each tumor and was used for statistical analysis. The prediction performance was assessed by the area under the receiver operating characteristic (ROC) curve (AUC), accuracy (ACC), sensitivity (SEN), specificity (SPE), positive predictive value (PPV), and negative predictive value (NPV). The AUCs between two models were statistically compared using a DeLong test (27). All statistical analyses were performed with $\mathrm{R}$ software, version 3.5.1 (https://www.rproject.org/). All statistical tests were two sided, and $p<0.05$ indicated significant. 


\section{RESULTS}

Patient and tumor characteristics are summarized in Table $\mathbf{1 .}$ No significant difference was found in patient age, histological grades, molecular subtypes and ALN status between the training and testing cohorts ( $p=0.457$ to 0.844 ).

\section{Image-Only Deep CNNs vs. Radiomics Models}

The predictive performance of the three image-only deep CNNs and the three image-only radiomics models in both training and testing cohorts is summarized in Table 2. Their ROC curves in both training and testing cohorts are shown in Figure 3, respectively. The radiomics feature selection results can be found in Table S3. Among all six image-only models, the combinedregion $\mathrm{CNN}$ achieved the best performance with a highest AUC of 0.912 and a highest accuracy of $89.3 \%$ in the testing cohort. In the testing cohort, the $\mathrm{CNN}$ built on each region performed better than the corresponding radiomics model built on the same region in terms of AUC and accuracy, but the differences of AUCs between the CNNs and their corresponding radiomics models were not statistically significant (Image-only CNN vs. Radiomics: Intratumoral: AUC 0.748 vs. $0.693, p=0.534$; Peritumoral: AUC 0.775 vs. $0.724, p=0.531$; Combined-region: AUC 0.912 vs. $0.886, p=0.601)$.

\section{Image-Molecular Deep CNNs vs. Radiomics Models}

The performance of the three image-molecular CNNs and the three image-molecular radiomics models is summarized in Table 3. Their ROC curves in both training and testing cohorts are shown in Figure 4. From Tables 2, 3, it can be found that the overall performance of the image-molecular models was slightly higher than those of their corresponding image-only models in the testing cohort, but no significant AUC differences were found between them. Among all 12 predictive models built in our study, the image-molecular CNN model built based on the combinedregion achieved the best performance with a highest AUC of 0.933 , a highest accuracy of $90.3 \%$ and a highest NPV of 0.958 in the testing cohort. All image-molecular CNNs achieved higher AUCs and higher accuracy than their corresponding radiomics models built based on the same tumoral region, but there were no significant differences between their AUCs (Image-molecular CNN vs. Radiomics: Intratumoral: AUC 0.794 vs. $0.706, p=$ 0.308 ; Peritumoral: AUC 0.813 vs. 0.743, $p=0.334$; Combinedregion: AUC 0.933 vs. $0.905, p=0.531$ ).

\section{Assessment of Peritumoral and Intratumoral Regions}

The predictive value of different tumoral regions were assessed by comparing the models built with the same machine learning methods (CNN or radiomics). It was observed that for the image-only CNNs and image-only radiomics models, the AUCs of the peritumoral models were slightly higher than those of the intratumoral models in the testing cohort, and their AUC differences were not significant (Image-only Peritumoral vs. Intratumoral: CNN: AUC 0.775 vs. $0.748, p=0.746$; Radiomics:
AUC 0.724 vs. $0.693, p=0.707)$. Similar results have been observed for the image-molecular models (Image-molecular Peritumoral vs. Intratumoral: CNN: AUC 0.813 vs. $0.794, p=$ 0.806; Radiomics: AUC 0.743 vs. 0.706, $p=0.647$ ).

The image-only CNNs and image-only radiomics models built based on combined-region achieved higher AUCs than their corresponding models built based on either the intratumoral or peritumoral region in the testing cohort, where the AUC differences between them were significant (Image-only Combined-region vs. [Peritumoral, Intratumoral]: CNN: AUC 0.912 vs. $[0.775,0.748],[p=0.049, p=0.031]$; Radiomics: AUC 0.886 vs. $[0.724,0.693],[p=0.014, p=0.004])$. The image-molecular CNNs and image-molecular radiomics models built based on combined-region also achieved higher AUCs. For image-molecular models, the difference between AUCs of the combined-region $\mathrm{CNN}$ and either the intratumoral $\mathrm{CNN}$ or peritumoral CNN was significant (Image-molecular Combinedregion vs. [Peritumoral, Intratumoral]: CNN: AUC 0.933 vs. [0.813, 0.794], $[p=0.048, p=0.046]$; Radiomics: AUC 0.905 vs. $[0.743,0.706],[p=0.006, p=0.003])$.

\section{Prospective Validation}

To further validate the CNNs and radiomics models, we performed a validation study using a relatively small prospective cohort. From November 182019 to December 12 2019, 16 breast cancer patients (6 node positive and 10 node negative) with 80 breast ultrasound images (each had 5 images as described in section Ultrasound Image Acquisition) were finally enrolled for analysis. Age, grade, and node status were obtained for the 16 patients and were summarized in Table 1. All six imageonly prediction models were tested. As we did not obtain IHC results, the image-molecular models were not tested. The model performance in this prospective cohort was summarized in Table 4. The ROC curves of all tested models were shown in Figure S2. We observed that the $\mathrm{CNN}$ built on the combined region achieved the highest AUC of 0.95 and the highest accuracy of $81.3 \%$, where two patients with positive node and one patient with negative node were misclassified. In general, CNNs outperformed radiomics models; prediction models built on combined region outperformed those built on either intratumor region or peritumor region only. The results were consistent with previous observation on the retrospective cohort.

\section{DISCUSSION}

The major findings of this study were that deep CNN, built by combining intratumoral and peritumoral regions in breast ultrasound images, outperformed radiomics models in predicting ALN metastasis. Although imaging-based machine learning approaches have been demonstrated useful in assessing breast cancers, few studies have been done on evaluating the value of intra- and peritumoral regions in metastasis prediction (9), and no studies have investigated how breast ultrasound-based deep CNNs performed compared with radiomics models. In this study, we first developed three types of CNN models based on intratumoral, peritumoral, and combined regions, respectively in ultrasound images for assessing the nodal 
TABLE 1 | A summary of patient and tumor characteristics of the study population.

\begin{tabular}{|c|c|c|c|c|c|c|}
\hline Variables & $\begin{array}{l}\text { All retrospective } \\
\text { patients }(n=479)\end{array}$ & $\begin{array}{c}\text { Training } \\
\text { cohort }(n=359)\end{array}$ & $\begin{array}{c}\text { Testing } \\
\text { cohort }(n=120)\end{array}$ & $P$-value & $\begin{array}{c}\text { Prospective } \\
\text { cohort }(n=16)\end{array}$ & $P$-value \\
\hline Age (mean \pm SD) & $48.7 \pm 11.1$ & $48.9 \pm 10.9$ & $47.9 \pm 11.9$ & 0.844 & $49.8 \pm 11.3$ & 0.680 \\
\hline I & 187 (39.0\%) & 140 (39.0\%) & 47 (39.2\%) & & $8(50.0 \%)$ & \\
\hline$\|$ & 249 (52.0\%) & 190 (52.9\%) & 59 (49.2\%) & & 7 (43.7\%) & \\
\hline Luminal A & 45 (9.4\%) & $33(9.2 \%)$ & 12 (10.0\%) & & - & \\
\hline Luminal B & $322(67.2 \%)$ & 239 (66.6\%) & $83(69.2 \%)$ & & - & \\
\hline HER2 positive & 57 (11.9\%) & 44 (12.3\%) & 13 (10.8\%) & & - & \\
\hline Triple negative & 55 (11.5\%) & 43 (11.9\%) & 12 (10.0\%) & & - & \\
\hline ALN & & & & 0.829 & & 0.418 \\
\hline
\end{tabular}

$P$-values were calculated by using $\chi^{2}$ test or Wilcoxon rank-sum test. $P$-values in the fifth column were calculated between training and testing cohorts. $P$-values in the seventh column were calculated between training and prospective cohorts.

TABLE 2 | A performance summary of the image-only CNNs and image-only radiomics models in training and testing cohorts in predicting ALN metastasis of breast cancer.

\begin{tabular}{|c|c|c|c|c|c|c|c|c|}
\hline \multicolumn{2}{|l|}{ Model } & \multirow{2}{*}{$\begin{array}{l}\text { Dataset } \\
\text { Training }\end{array}$} & \multirow{2}{*}{ 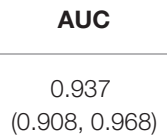 } & \multirow{2}{*}{$\begin{array}{c}\text { ACC (\%) } \\
84.6 \\
-\end{array}$} & \multirow{2}{*}{$\begin{array}{c}\text { SEN (\%) } \\
95.7 \\
(80.4,98.9)\end{array}$} & \multirow{2}{*}{$\begin{array}{c}\text { SPE (\%) } \\
80.3 \\
(76.6,95.4)\end{array}$} & \multirow{2}{*}{$\begin{array}{c}\text { PPV (\%) } \\
65.2 \\
(60.7,87.8)\end{array}$} & \multirow{2}{*}{$\begin{array}{c}\text { NPV (\%) } \\
98.0 \\
(92.5,99.5)\end{array}$} \\
\hline Image-only CNN & Intra & & & & & & & \\
\hline & Peri & Training & $\begin{array}{c}0.944 \\
(0.920,0.969)\end{array}$ & $\begin{array}{c}87.0 \\
-\end{array}$ & $\begin{array}{c}95.7 \\
(89.1,100.0)\end{array}$ & $\begin{array}{c}83.7 \\
(78.7,91.6)\end{array}$ & $\begin{array}{c}69.3 \\
(63.5,81.1)\end{array}$ & $\begin{array}{c}98.0 \\
(95.3,100.0)\end{array}$ \\
\hline & $\mathrm{Cmb}$ & Training & $\begin{array}{c}0.957 \\
(0.926,0.989)\end{array}$ & $\begin{array}{c}93.7 \\
-\end{array}$ & $\begin{array}{c}92.6 \\
(86.2,97.9)\end{array}$ & $\begin{array}{c}94.1 \\
(90.8,99.6)\end{array}$ & $\begin{array}{c}86.1 \\
(80.0,98.8)\end{array}$ & $\begin{array}{c}97.0 \\
(94.5,99.1)\end{array}$ \\
\hline & & Testing & $\begin{array}{c}0.912 \\
(0.834,99.0)\end{array}$ & $\begin{array}{c}89.3 \\
-\end{array}$ & $\begin{array}{c}85.7 \\
(67.9,96.4)\end{array}$ & $\begin{array}{c}90.7 \\
(84.0,100.0)\end{array}$ & $\begin{array}{c}77.4 \\
(66.7,100.0)\end{array}$ & $\begin{array}{c}94.4 \\
(89.0,98.6)\end{array}$ \\
\hline \multirow{4}{*}{$\begin{array}{l}\text { Image-only } \\
\text { radiomics }\end{array}$} & Peri & Training & $\begin{array}{c}0.920 \\
(0.882,0.958)\end{array}$ & $\begin{array}{c}87.3 \\
-\end{array}$ & $\begin{array}{c}82.6 \\
(72.8,93.5)\end{array}$ & $\begin{array}{c}89.1 \\
(80.3,96.7)\end{array}$ & $\begin{array}{c}74.5 \\
(63.6,89.9)\end{array}$ & $\begin{array}{c}93.0 \\
(90.2,97.0)\end{array}$ \\
\hline & & Testing & $\begin{array}{c}0.724 \\
(0.609,0.839)\end{array}$ & $\begin{array}{c}70.9 \\
-\end{array}$ & $\begin{array}{c}64.0 \\
(48.0,100.0)\end{array}$ & $\begin{array}{c}73.1 \\
(38.5,91.0)\end{array}$ & $\begin{array}{c}43.2 \\
(32.4,66.7)\end{array}$ & $\begin{array}{c}86.4 \\
(83.5,100.0)\end{array}$ \\
\hline & $\mathrm{Cmb}$ & Training & $\begin{array}{c}0.940 \\
(0.908,0.973)\end{array}$ & $\begin{array}{c}87.1 \\
-\end{array}$ & $\begin{array}{c}92.3 \\
(81.3,96.7)\end{array}$ & $\begin{array}{c}85.2 \\
(82.3,95.9)\end{array}$ & $\begin{array}{c}70.0 \\
(66.1,88.1)\end{array}$ & $\begin{array}{c}96.7 \\
(92.8,98.6)\end{array}$ \\
\hline & & Testing & $\begin{array}{c}0.886 \\
(0.831,0.942)\end{array}$ & $\begin{array}{c}83.3 \\
-\end{array}$ & $\begin{array}{c}87.5 \\
(72.5,97.5)\end{array}$ & $\begin{array}{c}81.8 \\
(75.5,91.8)\end{array}$ & $\begin{array}{c}63.6 \\
(55.7,78.7)\end{array}$ & $\begin{array}{c}94.7 \\
(90.0,98.9)\end{array}$ \\
\hline
\end{tabular}

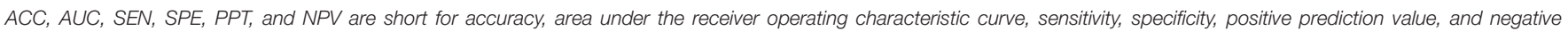

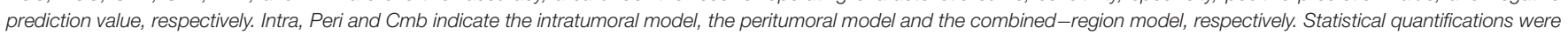
demonstrated with 95\% confidential interval (Cl), when applicable.

metastasis, and further compared the performance of the three CNNs with three radiomics models built based on the same regions in nodal metastasis prediction. Moreover, we evaluated if further benefit can be obtained by integrating ultrasound images and molecular subtype information into the predictive models. Note that besides a high AUC, a high NPV is also important as accurately identifying patients with negative nodes $[\sim 65 \%$ in all breast cancer patients (28)] 

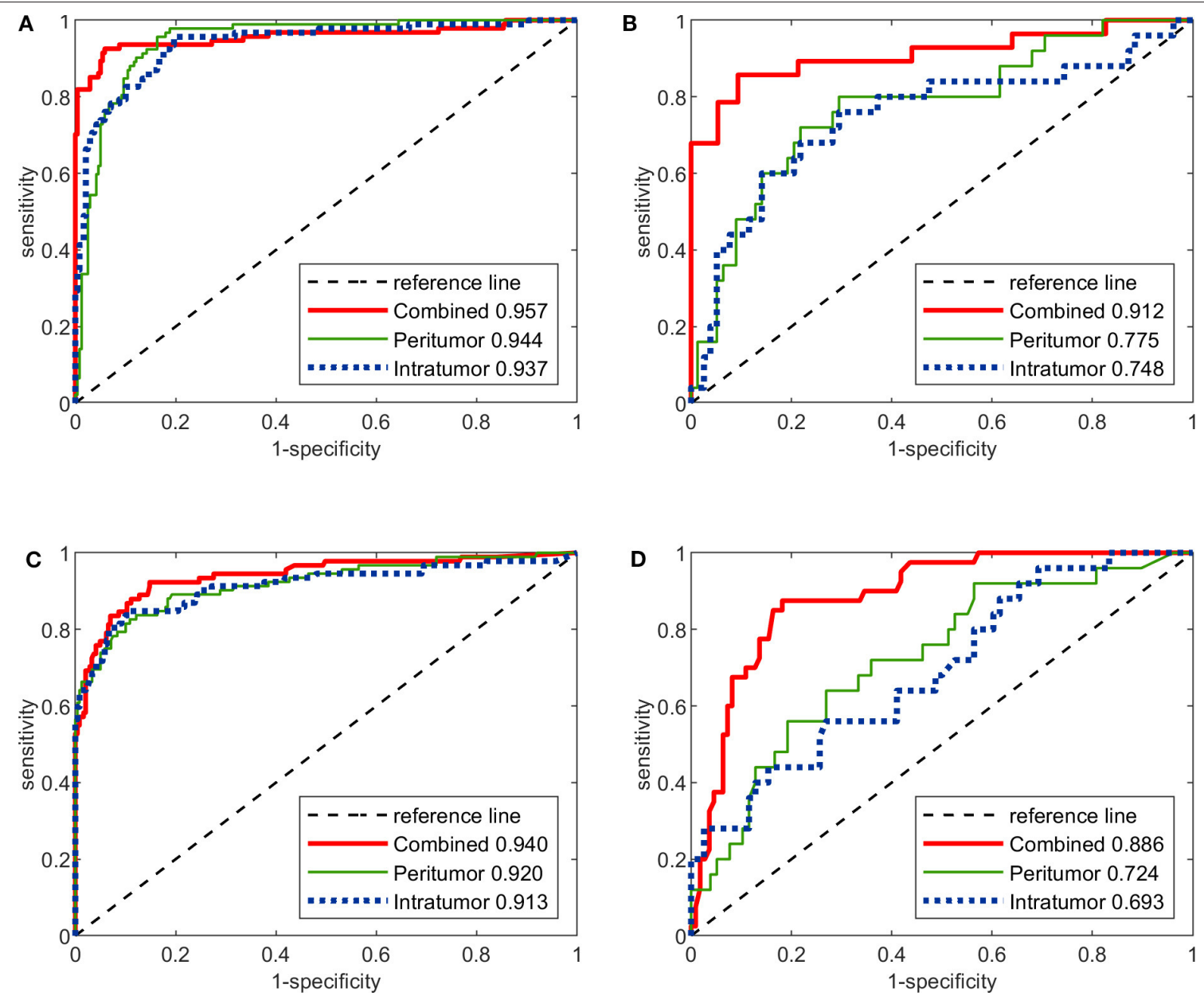

FIGURE 3 | The ROC curves of the three image-only deep CNNs and the three image-only radiomics models in both training and testing cohorts. (A) ROC curves of image-only CNNs in training cohort. (B) ROC curves of image-only CNNs in testing cohort. (C) ROC curves of image-only radiomics models in training cohort. (D) ROC curves of image-only radiomic models in testing cohort.

helps to avoid axillary overtreatment and reduce associated serious complications.

Identification of possible association between breast ultrasound features and ALN status has undoubtful clinical benefit. In clinical routine, the axilla can be staged clinically by palpation or surgically by SLNB or ALND. Although SLNB has less severe complications compared with ALND, it is not risk-free and SLNB- associated complications have been reported in large prospective trials (6). As palpation is inaccurate (29), AUS is performed to provide more relevant information. AUS alone has a reported sensitivity of $39-60 \%$, specificity of $90-96 \%$, PPV of $80-91 \%$, and NPV of $75-83 \%$ $(6,30,31)$. This implied that despite of an acceptable specificity above $90 \%$, prior to surgery about $40-60 \%$ of nodal metastases cannot be found by AUS and about $20-25 \%$ of patients with a negative AUS have been assessed as modal metastases after surgery. In case of suspicious ALN, AUS alone or combined with ultrasound-guided needle biopsy is performed for axillary staging to select patients who would benefit from ALND. A recent meta-analysis has shown that the use of AUS in stratifying patients directly to fast-track ALND without SLNB leads to overtreatment in up to two-thirds of patients (32). These data indicated that AUS alone is not sufficiently accurate for axillary staging.

Recent studies have shown the value of radiomics features from primary lesion in predicting the lymph node metastasis for different cancer sites, e.g., CT radiomics features in colorectal cancer (33), MRI/CT radiomics features in bladder cancer $(34,35)$ and esophageal cancer $(36)$. For breast cancer, two recent studies have assessed the value of radiomics features extracted from the primary tumor region at DCE-MRI and diffusion-weighted MRI (DWI) in predicting sentinel lymph node metastasis, where the reported AUC, sensitivity and specificity ranging from 0.805 to $0.869,0.700-0.778$, and $0.747-$ 861 respectively $(9,10)$. In our study, we built three image-only radiomics models by using both peri- and intratumoral regions in multiple ultrasound slices per lesion. The combined-region radiomics model achieved an AUC of 0.886 , a sensitivity of $87.5 \%$ 
TABLE 3 | A performance summary of the image-molecular CNNs and image-molecular radiomics models in training and testing cohorts in predicting ALN metastasis of breast cancer.

\begin{tabular}{|c|c|c|c|c|c|c|c|c|}
\hline Model & & Dataset & AUC & ACC (\%) & SEN (\%) & SPE (\%) & PPV (\%) & NPV (\%) \\
\hline \multirow[t]{6}{*}{$\begin{array}{l}\text { Image-molecular } \\
\text { CNN }\end{array}$} & Intra & Training & $\begin{array}{c}0.931 \\
(0.898,0.964)\end{array}$ & $\begin{array}{c}84.9 \\
-\end{array}$ & $\begin{array}{c}93.4 \\
(78.2,98.9)\end{array}$ & $\begin{array}{c}81.7 \\
(76.3,96.3)\end{array}$ & $\begin{array}{c}65.9 \\
(60.3,88.8)\end{array}$ & $\begin{array}{c}97.0 \\
(92.0,99.5)\end{array}$ \\
\hline & & Testing & $\begin{array}{c}0.794 \\
(0.677,0.911)\end{array}$ & $\begin{array}{c}72.8 \\
-\end{array}$ & $\begin{array}{c}80.0 \\
(48.0,96.0)\end{array}$ & $\begin{array}{c}70.5 \\
(60.3,100.0)\end{array}$ & $\begin{array}{c}46.5 \\
(40.3,100.0)\end{array}$ & $\begin{array}{c}91.7 \\
(84.5,98.0)\end{array}$ \\
\hline & Peri & Training & $\begin{array}{c}0.951 \\
(0.928,0.973)\end{array}$ & $\begin{array}{c}88.5 \\
-\end{array}$ & $\begin{array}{c}95.7 \\
(90.2,100.0)\end{array}$ & $\begin{array}{c}85.8 \\
(79.9,91.6)\end{array}$ & $\begin{array}{c}72.1 \\
(65.2,81.1)\end{array}$ & $\begin{array}{c}98.1 \\
(96.1,100.0)\end{array}$ \\
\hline & & Testing & $\begin{array}{c}0.813 \\
(0.717,0.909)\end{array}$ & $\begin{array}{c}75.7 \\
-\end{array}$ & $\begin{array}{c}88.0 \\
(64.0,100.0)\end{array}$ & $\begin{array}{c}71.8 \\
(64.1,92.3)\end{array}$ & $\begin{array}{c}50.0 \\
(43.1,74.1)\end{array}$ & $\begin{array}{c}94.9 \\
(88.2,100.0)\end{array}$ \\
\hline & $\mathrm{Cmb}$ & Training & $\begin{array}{c}0.962 \\
(0.933,0.990)\end{array}$ & $\begin{array}{c}92.8 \\
-\end{array}$ & $\begin{array}{c}93.5 \\
(83.9,97.8)\end{array}$ & $\begin{array}{c}92.5 \\
(89.6,100.0)\end{array}$ & $\begin{array}{c}82.9 \\
(77.9,100.0)\end{array}$ & $\begin{array}{c}97.4 \\
(94.1,99.1)\end{array}$ \\
\hline & & Testing & $\begin{array}{c}0.933 \\
(0.864,1.000)\end{array}$ & $\begin{array}{c}90.3 \\
-\end{array}$ & $\begin{array}{c}89.3 \\
(75.0,100.0)\end{array}$ & $\begin{array}{c}90.7 \\
(82.7,100.0)\end{array}$ & $\begin{array}{c}78.1 \\
(67.5,100.0)\end{array}$ & $\begin{array}{c}95.8 \\
(91.0,100.0)\end{array}$ \\
\hline \multirow[t]{6}{*}{$\begin{array}{l}\text { Image-molecular } \\
\text { radiomics }\end{array}$} & Intra & Training & $\begin{array}{c}0.931 \\
(0.898,0.964)\end{array}$ & $\begin{array}{c}85.8 \\
-\end{array}$ & $\begin{array}{c}89.0 \\
(78.0,95.6)\end{array}$ & $\begin{array}{c}84.6 \\
(78.7,94.6)\end{array}$ & $\begin{array}{c}68.6 \\
(61.5,84.8)\end{array}$ & $\begin{array}{c}95.3 \\
(91.7,98.1)\end{array}$ \\
\hline & & Testing & $\begin{array}{c}0.706 \\
(0.583,0.828)\end{array}$ & $\begin{array}{c}71.8 \\
-\end{array}$ & $\begin{array}{c}64.0 \\
(40.0,88.0)\end{array}$ & $\begin{array}{c}74.4 \\
(46.1,89.7)\end{array}$ & $\begin{array}{c}44.4 \\
(32.9,66.7)\end{array}$ & $\begin{array}{c}86.6 \\
(81.1,93.9)\end{array}$ \\
\hline & Peri & Training & $\begin{array}{c}0.916 \\
(0.877,0.955)\end{array}$ & $\begin{array}{c}88.2 \\
-\end{array}$ & $\begin{array}{c}84.8 \\
(76.1,91.3)\end{array}$ & $\begin{array}{c}89.5 \\
(85.8,95.4)\end{array}$ & $\begin{array}{c}75.7 \\
(70.0,87.2)\end{array}$ & $\begin{array}{c}93.9 \\
(90.9,96.5)\end{array}$ \\
\hline & & Testing & $\begin{array}{c}0.743 \\
(0.640,0.847)\end{array}$ & $\begin{array}{c}71.8 \\
-\end{array}$ & $\begin{array}{c}72.0 \\
(60.0,100.0)\end{array}$ & $\begin{array}{c}71.8 \\
(39.7,85.9)\end{array}$ & $\begin{array}{c}45.0 \\
(33.8,60.7)\end{array}$ & $\begin{array}{c}88.9 \\
(86.1,100.0)\end{array}$ \\
\hline & $\mathrm{Cmb}$ & Training & $\begin{array}{c}0.950 \\
(0.921,0.980)\end{array}$ & $\begin{array}{c}90.1 \\
-\end{array}$ & $\begin{array}{c}89.0 \\
(81.3,96.7)\end{array}$ & $\begin{array}{c}90.5 \\
(81.5,96.7)\end{array}$ & $\begin{array}{c}77.9 \\
(65.9,90.4)\end{array}$ & $\begin{array}{c}95.7 \\
(93.1,98.7)\end{array}$ \\
\hline & & Testing & $\begin{array}{c}0.905 \\
(0.855,0.956)\end{array}$ & $\begin{array}{c}84.0 \\
-\end{array}$ & $\begin{array}{c}90.0 \\
(77.5,97.5)\end{array}$ & $\begin{array}{c}81.8 \\
(74.5,93.6)\end{array}$ & $\begin{array}{c}64.3 \\
(56.5,82.9)\end{array}$ & $\begin{array}{c}95.7 \\
(91.2,98.9)\end{array}$ \\
\hline
\end{tabular}

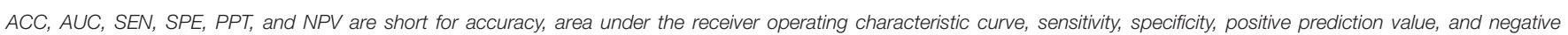

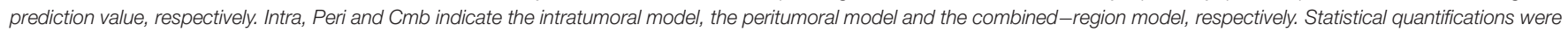
demonstrated with 95\% confidential interval (Cl), when applicable.

and a specificity of $81.8 \%$ on the testing cohort, which were comparable with the previous radiomics models built with MRI.

Although promising, an efficient radiomics analysis heavily relies on a handcrafted image processing pipeline comprising three tightly coupled steps: feature extraction, feature selection and machine learning model building. Small variations in each stage may affect the prediction accuracy and stability (37). Deep $\mathrm{CNN}$ improves this pipeline by automatically learning predictive features on its own and yields a class probability vector as output. Currently, CNN-based learning methods have achieved diagnostic accuracy levels in skin cancer (11) and retinal diseases $(12,13)$, which have been unattainable via radiomics approaches. For breast cancer, a comparative study (38) demonstrated that $\mathrm{CNN}$ was superior to radiomic analysis in terms of a significantly higher AUC (0.88 vs. 0.81, $p<0.001)$ for classification of enhancing lesions as benign or malignant at MRI. Another comparative study in Kooi et al. (39) also demonstrated that $\mathrm{CNN}$ was superior to radiomics-based software in detection of mammographic breast lesions. In our study, all six CNNs (three image-only and three image-molecular) achieved higher AUC and accuracy than corresponding radiomics models built on the same regions on both training and testing cohorts. Note that in our results the differences between their AUCs (CNN vs. radiomics) were not significant (DeLong $p>0.05$ ).

Most image analysis studies on breast cancer was focused on the intratumoral region. Evidences have demonstrated that imaging features of peritumoral regions can offer outcomerelated information. Several studies have demonstrated that the enhancement patterns of tumor-adjacent parenchyma in DCEMRI were associated with chemotherapy response (14), local recurrence (15), and survival (16) in breast cancer. In a recent study (40) the grade of peritumoral edema identified in breast MRI has been independently associated with disease recurrence. In study by Zhou et al. (41), it was demonstrated that the peritumoral stiffness assessed by ultrasound elastography of malignant breast lesions was higher than that of benign lesions. A 2017 study (17) was the first attempt to extract radiomics features from both intratumoral and peritumoral regions in breast DCE-MRI, where the features successfully predicted the pathological complete response to neoadjuvant chemotherapy. A more recent 2019 study (9) for the first time demonstrated the feasibility of predicting sentinel lymph node metastasis by using intratumoral and peritumoral radiomics features in DCE-MRI, achieving an AUC of 0.806 and an NPV of $82.4 \%$ with radiomics features only. Our study has shown the value of peritumoral ultrasonographic $\mathrm{CNN}$ features in predicting nodal metastasis with an AUC of 0.775 and an NPV of $91.6 \%$. By combining both intra- and peritumoral regions, the CNN achieved a significantly better AUC of 0.912 and an NPV of $94.4 \%$. The FNRs of the image-only CNN model built by combining the intra- and peritumoral regions achieved 5.9, 9.3, and $10 \%$ in the training, testing, and prospective data sets, 

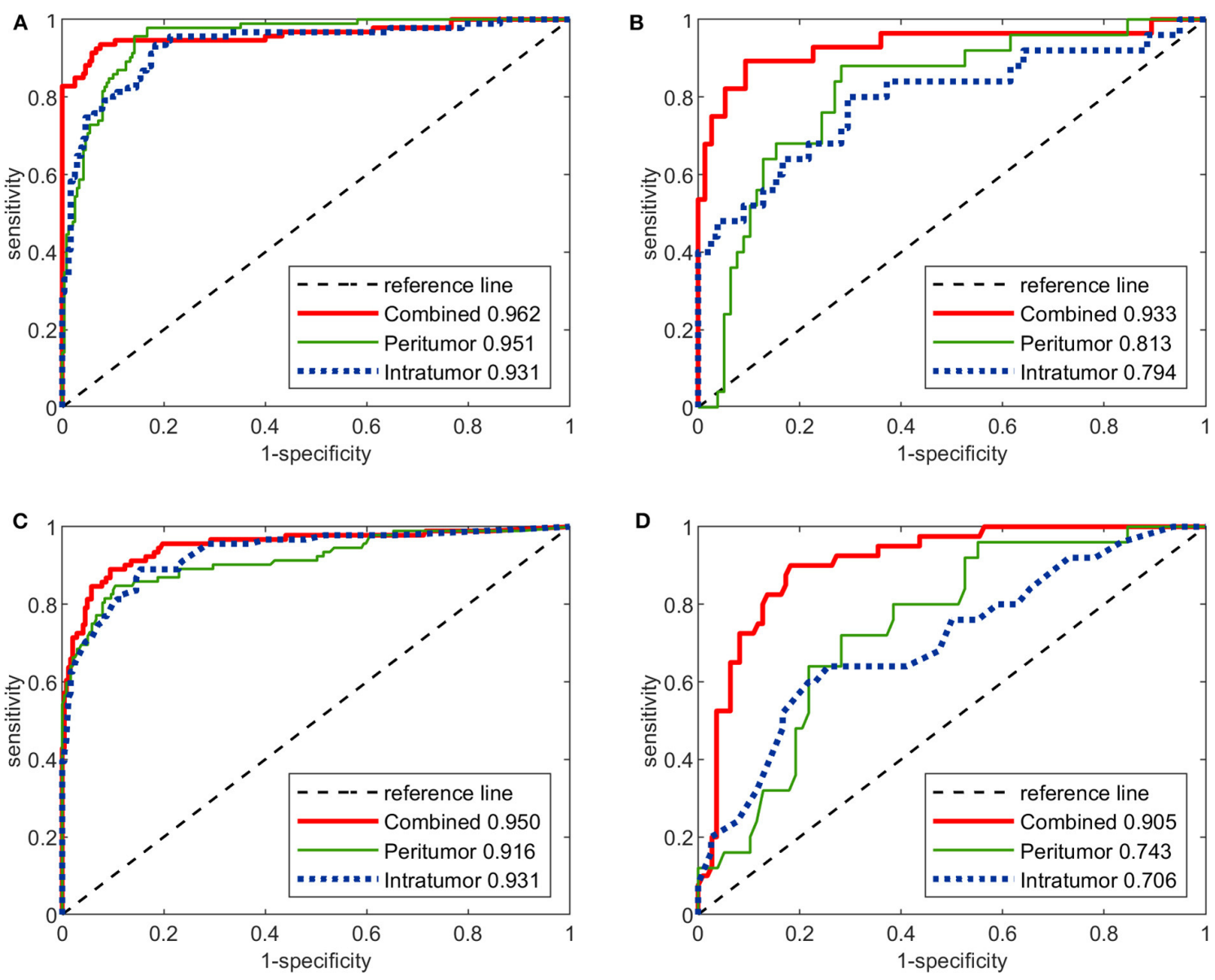

FIGURE 4 | The ROC curves of the three image-molecular deep CNNs and the three image-molecular radiomics models in both training and testing cohorts. (A) ROC curves of image-molecular CNNs in training cohort. (B) ROC curves of image-molecular CNNs in testing cohort. (C) ROC curves of image-molecular radiomics models in training cohort. (D) ROC curves of image-molecular radiomic models in testing cohort.

TABLE 4 | A performance summary of the image-only CNNs and image-only radiomics models in the prospective cohorts in predicting ALN metastasis of breast cancer.

\begin{tabular}{|c|c|c|c|c|c|c|c|}
\hline Model & & AUC & ACC (\%) & SEN (\%) & SPE (\%) & PPV (\%) & NPV (\%) \\
\hline \multirow[t]{3}{*}{ Image-only CNN } & Intra & 0.767 & 75.6 & 50.0 & 90.0 & 75.0 & 75.0 \\
\hline & Peri & 0.850 & 75.0 & 50.0 & 90.0 & 75.0 & 75.0 \\
\hline & $\mathrm{Cmb}$ & 0.950 & 81.3 & 66.7 & 90.0 & 80.0 & 81.8 \\
\hline \multirow{3}{*}{$\begin{array}{l}\text { Image-only } \\
\text { radiomics }\end{array}$} & Intra & 0.533 & 68.8 & 33.3 & 90.0 & 66.7 & 69.2 \\
\hline & Peri & 0.533 & 68.8 & 33.3 & 90.0 & 66.7 & 69.2 \\
\hline & $\mathrm{Cmb}$ & 0.833 & 81.3 & 83.3 & 80.0 & 71.4 & 88.9 \\
\hline
\end{tabular}

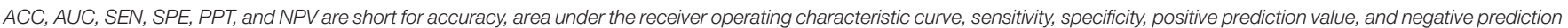
value, respectively. Intra, Peri and $\mathrm{Cmb}$ indicate the intratumoral model, the peritumoral model and the combined-region model, respectively.

respectively, which were superior to the image-only radiomics model with FNRs of $14.8,18.25$, and $20 \%$ in the training, testing, and prospective data sets, respectively. The FNRs of the CNN model were comparable with those of SLNB [4.6 to $16.7 \%(4)$ ] and were higher than the radiomics models $[13.9$ to $25 \%(9,10)]$ reported previously. By integrating the molecular subtype information, all the obtained image-molecular models, either CNN or radiomics, achieved slighter higher AUCs and NPVs.

The biological mechanism underlying the peritumoral imaging features and their association with clinical outcomes remains unclear. Many cancer studies have shown that biological changes in the tissue immediately surrounding the breast tumor mass might be potential predictive or prognostic 
markers, such as peritumor lymphovascular invasion $(42,43)$, peritumoral lymphocytic infiltration (44), and peritumoral edema (45). In study by Zhao et al. (46) it was suggested that vascular endothelial growth factor (VEGF)-C/D induced peritumoral lymphangiogenesis may be one mechanism that leads to metastatic spread. In study by $\mathrm{Wu}$ et al. (16) the prognostic peritumoral features were associated with the tumor necrosis factor (TNF) signaling pathway that has been involved in oncogenic angiogenesis, invasion, and metastasis (47). Further studies are warranted to determine how the underlying biological changes were reflected by peritumor imaging features.

Our study has several limitations. The first limitation was the limited population size which may lead to bias. Larger patient population from more centers should be involved in future to improve the machined learning-based models. The population size of the prospective cohort is particularly small, where significant bias may occur. We will recruit more prospective data in future to further evaluate our methods in clinical practice. The second limitation was that all image data was obtained on the same type of ultrasound machine. In future we will evaluate our models on more heterogeneous image data acquired with different machines. Moreover, we built our CNNs and radiomics models using only ultrasound images and molecular subtypes. In future we will build more comprehensive models by incorporating more clinical and pathological data. Our future research also includes the exploring of biological mechanism underlying the association between intratumoral/peritumoral imaging features and nodal metastasis. We will also assess the possible incremental value of the tumoral ultrasonographic features over the AUS in axillary staging.

In conclusion, CNNs built on tumoral regions in ultrasound images allowed accurate prediction of ALN metastasis, which achieved higher AUC and NPV than radiomics models. Either CNNs or radiomic models built on peritumor regions performed slighter better than those built on intratumor regions, while combining both intra- and peritumoral regions achieved significantly better AUCs and higher NPVs. Further

\section{REFERENCES}

1. Segal R, Miller K, Jemal A. Cancer statistics, 2018. CA Cancer J Clin. (2018) 68:7-30. doi: 10.3322/caac.21442

2. Giuliano AE, Connolly JL, Edge SB, Mittendorf EA, Rugo HS, Solin LJ, et al. Breast cancer-major changes in the American Joint Committee on Cancer eighth edition cancer staging manual. Cancer J Clin. (2017) 67:290-303. doi: $10.3322 /$ caac. 21393

3. Giuliano AE, Hunt KK, Ballman KV, Beitsch PD, Whitworth PW, Blumencranz PW, et al. Axillary dissection vs. no axillary dissection in women with invasive breast cancer and sentinel node metastasis: a randomized clinical trial. JAMA. (2011) 305:569-75. doi: 10.1001/jama.2 011.90

4. Lyman GH, Temin S, Edge SB, Newman LA, Turner RR, Weaver DL, et al. Sentinel lymph node biopsy for patients with early-stage breast cancer: American Society of Clinical Oncology clinical practice guideline update. $J$ Clin Oncol. (2014) 32:1365-83. doi: 10.1200/JCO.2013.54.1177 integrating the molecular subtype information into either CNNs or radiomics models can slightly benefit the performance.

\section{DATA AVAILABILITY STATEMENT}

To achieve repeatability, the data set of this study, including pretrained CNN models, imaging data of the prospective cohort, statistical analysis, and the Python implementation, was deposited into the Mendeley data library (https://data.mendeley. $\mathrm{com} /$ datasets/rc32mg38rb/draft?a=2333e5fd-e7b1-4603-b06e-

b609d79bab11).

\section{ETHICS STATEMENT}

The studies involving human participants were reviewed and approved by Ethics Committee of Peking University Shenzhen Hospital. The ethics committee waived the requirement of written informed consent for participation.

\section{AUTHOR CONTRIBUTIONS}

DS, Z-CL, and DL conceived and designed the study. $\mathrm{XL}$ collected the clinical and image data and performed image pre-processing. QS, YZ, LL, and KY analyzed the image data and performed the statistical analysis. QS wrote the manuscript. All authors approved the final manuscript.

\section{FUNDING}

This work was supported by the National Natural Science Foundation of China (no. 61571432) and Shenzhen Basic Research Program (JCYJ20170413162354654).

\section{SUPPLEMENTARY MATERIAL}

The Supplementary Material for this article can be found online at: https://www.frontiersin.org/articles/10.3389/fonc. 2020.00053/full\#supplementary-material

5. Lucci A, McCall LM, Beitsch PD, Whitworth PW, Reintgen DS, Blumencranz PW, et al. Surgical complications associated with sentinel lymph node dissection (SLND) plus axillary lymph node dissection compared with SLND alone in the American College of Surgeons Oncology Group Trial Z0011. J Clin Oncol. (2007) 25:3657-63. doi: 10.1200/JCO.2006.0 7.4062

6. Wilke LG, McCall LM, Posther KE, Whitworth PW, Reintgen DS, Leitch AM, et al. Surgical complications associated with sentinel lymph node biopsy: results from a prospective international cooperative group trial. Ann Surg Oncol. (2006) 13:491-500. doi: 10.1245/ASO.2006. 05.013

7. Feng Y, Huang R, He Y, Lu A, Fan Z, Fan T, et al. Efficacy of physical examination, ultrasound, and ultrasound combined with fine-needle aspiration for axilla staging of primary breast cancer. Breast Cancer Res Treat. (2015) 149:761-5. doi: 10.1007/s10549-015-3280-z

8. Ahmed M, Douek M. Is axillary ultrasound imaging necessary for all patients with breast cancer? Br J Surg. (2018) 105:930-2. doi: 10.1002/bjs.10784 
9. Liu C, Ding J, Spuhler K, Gao Y, Serrano Sosa M, Moriarty M, et al. Preoperative prediction of sentinel lymph node metastasis in breast cancer by radiomic signatures from dynamic contrast-enhanced MRI. J Magnet Reson Imaging. (2019) 49:131-40. doi: 10.1002/jmri. 26224

10. Dong Y, Feng Q, Yang W, Lu Z, Deng C, Zhang L, et al. Preoperative prediction of sentinel lymph node metastasis in breast cancer based on radiomics of T2-weighted fat-suppression and diffusion-weighted MRI. Eur Radiol. (2018) 28:582-91. doi: 10.1007/s00330-017-5005-7

11. Esteva A, Kuprel B, Novoa RA, Ko J, Swetter SM, Blau HM, et al. Dermatologist-level classification of skin cancer with deep neural networks. Nature. (2017) 542:115. doi: 10.1038/nature21056

12. Kermany DS, Goldbaum M, Cai W, Valentim CC, Liang H, Baxter SL, et al. Identifying medical diagnoses and treatable diseases by image-based deep learning. Cell. (2018) 172:1122-31. e1129. doi: 10.1016/j.cell.2018. 02.010

13. De Fauw J, Ledsam JR, Romera-Paredes B, Nikolov S, Tomasev N, Blackwell $\mathrm{S}$, et al. Clinically applicable deep learning for diagnosis and referral in retinal disease. Nat Med. (2018) 24:1342. doi: 10.1038/s41591-018-0107-6

14. Hattangadi J, Park C, Rembert J, Klifa C, Hwang J, Gibbs J, et al. Breast stromal enhancement on MRI is associated with response to neoadjuvant chemotherapy. Am J Roentgenol. (2008) 190:1630-6. doi: 10.2214/AJR.0 7.2533

15. Kim S-A, Cho N, Ryu EB, Seo M, Bae MS, Chang JM, et al. Background parenchymal signal enhancement ratio at preoperative MR imaging: association with subsequent local recurrence in patients with ductal carcinoma in situ after breast conservation surgery. Radiology. (2013) 270:699-707. doi: 10.1148/radiol.13130459

16. Wu J, Li B, Sun X, Cao G, Rubin DL, Napel S, et al. Heterogeneous enhancement patterns of tumor-adjacent parenchyma at MR imaging are associated with dysregulated signaling pathways and poor survival in breast cancer. Radiology. (2017) 285:401-13. doi: 10.1148/radiol.20171 62823

17. Braman NM, Etesami M, Prasanna P, Dubchuk C, Gilmore H, Tiwari P, et al. Intratumoral and peritumoral radiomics for the pretreatment prediction of pathological complete response to neoadjuvant chemotherapy based on breast DCE-MRI. Breast Cancer Res. (2017) 19:57. doi: 10.1186/s13058-01 7-0862-1

18. Curigliano G, Burstein HJP, Winer E, Gnant M, Dubsky P, Loibl S, et al. De-escalating and escalating treatments for early-stage breast cancer: the St. Gallen International Expert Consensus Conference on the Primary Therapy of Early Breast Cancer 2017. Ann Oncol. (2017) 28:1700-12. doi: 10.1093/annonc/mdx308

19. Sickles EA, D’Orsi CJ, Bassett LW, Appleton CM, Berg WA, Burnside ES. ACR BI-RADS ${ }^{\circledR}$ Atlas, Breast imaging reporting and data system. Reston, VA: American College of Radiology (2013). P. 39-48.

20. Huang G, Liu Z, Van Der Maaten L, Weinberger KQ. Densely connected convolutional networks. In: Proceedings of the IEEE Conference on Computer Vision and Pattern Recognition. Honolulu, HI (2017).

21. Van Griethuysen JJ, Fedorov A, Parmar C, Hosny A, Aucoin N, Narayan V, et al. Computational radiomics system to decode the radiographic phenotype. Cancer Res. (2017) 77:e104-7. doi: 10.1158/0008-5472.CAN-17-0339

22. Aerts HJ, Velazquez ER, Leijenaar RT, Parmar C, Grossmann P, Carvalho S, et al. Decoding tumour phenotype by noninvasive imaging using a quantitative radiomics approach. Nat Commun. (2014) 5:4006. doi: 10.1038/ncomms5006

23. Lambin P, Leijenaar RT, Deist TM, Peerlings J, De Jong EE, Van Timmeren J, et al. Radiomics: the bridge between medical imaging and personalized medicine. Nat Rev Clin Oncol. (2017) 14:749. doi: 10.1038/nrclinonc.20 17.141

24. Kursa MB, Rudnicki WR. Feature selection with the Boruta package. J Stat Softw. (2010) 36:1-13. doi: 10.18637/jss.v036.i11

25. Breiman L. Random forests. Mach Learn. (2001) 45:5-32. doi: 10.1023/A:1010933404324

26. Louppe G, Wehenkel L, Sutera A, Geurts P. Understanding variable importances in forests of randomized trees. Adv Neural Informat Process Syst. (2013) 1:431-9.
27. DeLong ER, DeLong DM, Clarke-Pearson DL. Comparing the areas under two or more correlated receiver operating characteristic curves: a nonparametric approach. Biometrics. (1988) 44:837-45. doi: 10.2307/25 31595

28. Kuijs V, Moossdorff M, Schipper R, Beets-Tan R, Heuts E, Keymeulen $\mathrm{K}$, et al. The role of MRI in axillary lymph node imaging in breast cancer patients: a systematic review. Insights Into Imaging. (2015) 6:203-15. doi: 10.1007/s13244-015-0404-2

29. Lanng C, Hoffmann J, Galatius H, Engel U. Assessment of clinical palpation of the axilla as a criterion for performing the sentinel node procedure in breast cancer. Eur J Surg Oncol. (2007) 33:281-4. doi: 10.1016/j.ejso.2006. 09.032

30. Bailey A, Layne G, Shahan C, Zhang J, Wen S, Radis S, et al. Comparison between ultrasound and pathologic status of axillary lymph nodes in clinically node-negative breast cancer patients. Am Surg. (2015) 81:865-9.

31. Helfgott R, Mittlboeck M, Miesbauer M, Moinfar F, Haim S, Mascherbauer M, et al. The influence of breast cancer subtypes on axillary ultrasound accuracy: a retrospective single center analysis of 583 women. Eur J Surg Oncol. (2018) 45:538-43. doi: 10.1016/j.ejso.2018.10.001

32. Ahmed M, Jozsa F, Baker R, Rubio I, Benson J, Douek M. Meta-analysis of tumour burden in pre-operative axillary ultrasound positive and negative breast cancer patients. Breast Cancer Res Treatment. (2017) 166:329-36. doi: 10.1007/s10549-017-4405-3

33. Huang Y, Liang C, He L, Tian J, Liang C, Chen X, et al. Development and validation of a radiomics nomogram for preoperative prediction of lymph node metastasis in colorectal cancer. J Clin Oncol. (2016) 34:2157-64. doi: 10.1200/JCO.2015.65.9128

34. Wu S, Zheng J, Li Y, Yu H, Shi S, Xie W, et al. A radiomics nomogram for the preoperative prediction of lymph node metastasis in bladder cancer. Clin Cancer Res. (2017) 23:6904-11. doi: 10.1158/1078-0432.CCR17-1510

35. Wu S, Zheng J, Li Y, Wu Z, Shi S, Huang M, et al. Development and validation of an MRI-based radiomics signature for the preoperative prediction of lymph node metastasis in bladder cancer. EBioMedicine. (2018) 34:76-84. doi: 10.1016/j.ebiom.2018.07.029

36. Qu J, Shen C, Qin J, Wang Z, Liu Z, Guo J, et al. The MR radiomic signature can predict preoperative lymph node metastasis in patients with esophageal cancer. Eur Radiol. (2019) 29:906-14. doi: 10.1007/s00330-0185583-z

37. Li Q, Bai H, Chen Y, Sun Q, Liu L, Zhou S, et al. A fully-automatic multiparametric radiomics model: towards reproducible and prognostic imaging signature for prediction of overall survival in glioblastoma multiforme. Sci Rep. (2017) 7:14331. doi: 10.1038/s41598-01714753-7

38. Truhn D, Schrading S, Haarburger C, Schneider H, Merhof D, Kuhl C. Radiomic versus convolutional neural networks analysis for classification of contrast-enhancing lesions at multiparametric breast, MRI. Radiology. (2018) 290:290-7. doi: 10.1148/radiol.2018181352

39. Kooi T, Litjens G, Van Ginneken B, Gubern-Mérida A, Sánchez CI, Mann R, et al. Large scale deep learning for computer aided detection of mammographic lesions. Med Image Anal. (2017) 35:303-12. doi: 10.1016/j.media.2016.07.007

40. Cheon H, Kim HJ, Kim TH, Ryeom H-K, Lee J, Kim GC, et al. Invasive breast cancer: Prognostic value of peritumoral edema identified at preoperative MR imaging. Radiology. (2018) 287:68-75. doi: 10.1148/radiol.20171 71157

41. Zhou J, Zhan W, Dong Y, Yang Z, Zhou C. Stiffness of the surrounding tissue of breast lesions evaluated by ultrasound elastography. Eur Radiol. (2014) 24:1659-67. doi: 10.1007/s00330-014-3152-7

42. Schoppmann SF, Bayer G, Aumayr K, Taucher S, Geleff S, Rudas M, et al. Prognostic value of lymphangiogenesis and lymphovascular invasion in invasive breast cancer. Ann Surg. (2004) 240:306-12. doi: 10.1097/01.sla.0000133355.48672.22

43. Ejlertsen B, Jensen M-B, Rank F, Rasmussen BB, Christiansen P, Kroman N, et al. Population-based study of peritumoral lymphovascular invasion and outcome among patients with operable breast cancer. J Natl Cancer Inst. (2009) 101:729-35. doi: 10.1093/jnci/djp090 
44. Ocaña A, Diez-Gónzález L, Adrover E, Fernández-Aramburo A, Pandiella A, Amir E. Tumor-infiltrating lymphocytes in breast cancer: ready for prime time? J Clin Oncol. (2015) 33:1298-9. doi: 10.1200/JCO.2014.59.7286

45. Uematsu T. Focal breast edema associated with malignancy on T2weighted images of breast MRI: peritumoral edema, prepectoral edema, and subcutaneous edema. Breast Cancer. (2015) 22:66-70. doi: 10.1007/s12282-014-0572-9

46. Zhao Y-C, Ni X-J, Li Y, Dai M, Yuan Z-X, Zhu Y-Y, et al. Peritumoral lymphangiogenesis induced by vascular endothelial growth factor $\mathrm{C}$ and $\mathrm{D}$ promotes lymph node metastasis in breast cancer patients. World J Surg Oncol. (2012) 10:165. doi: 10.1186/1477-7819-10-165

47. Balkwill F. TNF- $\alpha$ in promotion and progression of cancer. Cancer Metastasis Rev. (2006) 25:409. doi: 10.1007/s10555-006-9005-3
Conflict of Interest: LL was employed by the company Ultimage Lab.

The remaining authors declare that the research was conducted in the absence of any commercial or financial relationships that could be construed as a potential conflict of interest.

Copyright $\odot 2020$ Sun, Lin, Zhao, Li, Yan, Liang, Sun and Li. This is an open-access article distributed under the terms of the Creative Commons Attribution License (CC $B Y)$. The use, distribution or reproduction in other forums is permitted, provided the original author(s) and the copyright owner(s) are credited and that the original publication in this journal is cited, in accordance with accepted academic practice. No use, distribution or reproduction is permitted which does not comply with these terms. 